\title{
VALIDACIÓN DE UN CUESTIONARIO DE CULTURA ERGONÓMICA EN CENTROS DE TRABAJO CCE-T
}

Claudia Patricia Ardila-Jaimes, FT M.Sc., Reynaldo Mauricio Rodríguez-Amaya, MD M.Sc. ${ }^{1}$

\section{Resumen}

Objetivo. Diseñar y validar un cuestionario de cultura ergonómica en centros de trabajo (CCE-T). Métodos. Se realizó un estudio de evaluación de pruebas diagnósticas entre junio de 2015 y diciembre de 2016 en las siguientes fases: 1. Revisión de la literatura; 2. Recuento de los constructos que conforman las dimensiones macroergonomía y microergonomía; 3. Diseño del cuestionario con respuestas tipo Likert de 27 ítems; 4 . Validez de contenido con grupo de 5 expertos; 5. Validez facial con 16 sujetos; y 6 . Prueba de validez y confiabilidad a 162 sujetos. Resultados. El CCE-T mostró un alfa de Cronbach de $a=0,896$. Este resultado, a la luz de la intención de esta validación, se clasifica en una confiabilidad muy alta.Conclusion. El CCE-T muestra ser un instrumento con una validez, confiabilidad y utilidad aceptables para medir el nivel de desarrollo de la cultura ergonómica en centros de trabajo, desde la óptica del trabajador.

${ }^{1}$ Docentes investigadores Grupo de Investigación en Salud, Rehabilitación y Trabajo SARET. Universidad Manuela Beltrán Seccional Bucaramanga. Correspondencia a: Calle 33 No. 27-33, Bucaramanga, Colombia. E-mail: claudia. ardila@outlook.com 


\title{
VALIDATION OF AN ERGONOMIC CULTURE QUESTIONNAIRE IN CCE-T WORK CENTERS
}

Claudia Patricia Ardila-Jaimes, FT M.Sc., Reynaldo Mauricio Rodríguez-Amaya, MD M.Sc. ${ }^{1}$

\begin{abstract}
Objective. Design and validate an Ergonomic Culture questionnaire in work centers. Methods. A study to evaluate diagnostic tests was conducted between June 2015 and December 2016 in the following phases: 1) Literature Review 2) Review the constructs which stablished the Macroergonomy and Macroergonomy dimensions 3) Design the questionnaire based on Likert type responses with 27 items 4) Content validation with a group of 5 experts 5) Facial validity with 16 subjects and 6) Validity and reliability test applied to 162 subjects. Results. The CCE-T showed an Alpha of Cronbach of $\alpha=0,896$, this result, in the light of the purpose of this validation process, is classified as Very High reliability. Conclusion. The CCE-T shows to be an instrument of acceptable validity, reliability, and usefulness to measure the level of Ergonomic Culture development from the perspective of the worker in work centers.
\end{abstract}




\section{VALIDAÇ̃̃O DE UM QUESTIONÁRIO PARA CULTURA ERGONỐMICA EM CENTROS DE TRABALHO CCE-T}

Claudia Patricia Ardila-Jaimes, FT M.Sc., Reynaldo Mauricio Rodríguez-Amaya, MD M.Sc. ${ }^{1}$

\section{Abstract}

Objetivo. Elaborar e validar um questionário de cultura ergonômica em centros de trabalho. Métodos. Foi realizado um estudo de avaliação dos testes de diagnóstico, entre Junho de 2015 e Dezembro de 2016 nas seguintes fases: 1) revisão da literatura 2) contagem dos construtos que compõem as dimensões Macroergonomia e Macroergonomia 3) desenho do questionário com respostas tipo Likert de 27 itens 4) validade do conteúdo com um grupo de 5 peritos 5) validade aparente com 16 indivíduos e 6) teste de validade e de fiabilidade para 162 indivíduos. Resultados. O CCE-T apresentou alfa de Cronbach $\alpha=0,896$, este resultado, tendo em conta a intenção desta validação, é classificada como uma fiabilidade muito elevada. Conclusão. O CCE-T é um instrumento com validade, fiabilidade e utilidade aceitáveis para medir o nível de desenvolvimento da cultura ergonômica em centros de trabalho a partir do ponto de vista dos trabalhadores. 


\section{Introducción}

Los desórdenes musculoesqueléticos (DME) de origen ocupacional son una problemática mundial que afecta tanto a países desarrollados como a aquellos en vía de desarrollo; son considerados la primera causa de enfermedad en el trabajo en la Unión Europea(1) y en el año 2016, los DME representaron más del $41 \%$ de todas las enfermedades laborales en el Reino Unido(2) y casi el 66\% de todas las enfermedades laborales en Corea del Sur(3). A su vez, representaron el $40 \%$ de los costos de salud relacionados con el trabajo en todo el mundo(4). Colombia, por su parte, refiere un comportamiento similar; por ejemplo, en el año 2012 , el $88 \%$ de las enfermedades calificadas como de origen ocupacional fueron DME (5), entre ellas se encuentran el síndrome del túnel carpiano y las tendinitis en miembros superiores, razón por la que representan una alta carga para los sistemas de salud y riesgos laborales.

Existen factores individuales y psicosociales asociados al desarrollo de DME de origen ocupacional; sin embargo, son tal vez los factores físicos los que más influyen en su aparición, principalmente por la manipulación de cargas, los movimientos repetitivos de los miembros superiores, la vibración, el frío y las posturas forza- das(1). Algunas revisiones han aportado datos sobre los beneficios que conllevan las intervenciones en ergonomía a dichos factores, subrayando la importancia de incluir en estas estrategias de intervención, no solo a los trabajadores, sino a los supervisores y a la alta dirección(6). Dicho esto, se espera entonces que una empresa desarrolle un alto grado de cultura ergonómica, como piedra angular de lo que se considera la intervención primaria en ergonomía(7) y como una base del éxito en los programas de salud colectiva que allí puedan desarrollarse.

El término cultura ergonómica se entiende como "el conjunto de formas y expresiones aplicadas para trasformar el trabajo, que caracterizan a una organización" por tanto, para que exista una verdadera cultura ergonómica se requiere, entre otros, de un "ambiente favorable, un pensar colectivo y de la reafirmación social de las necesidades en los lugares de trabajo"(8). Para determinar las dimensiones de la cultura ergonómica, se revisó el modelo de aplicación de la ergonomía de Scott, Kogi y McPhee(9) (ver Figura 1), cuyos componentes se proponen como pilares en el desarrollo de una cultura ergonómica en el lugar de trabajo. Describen desde las actividades organizativas (macroergonomía), hasta el estudio de la interfaz hombre-máquina (microergonomía). 


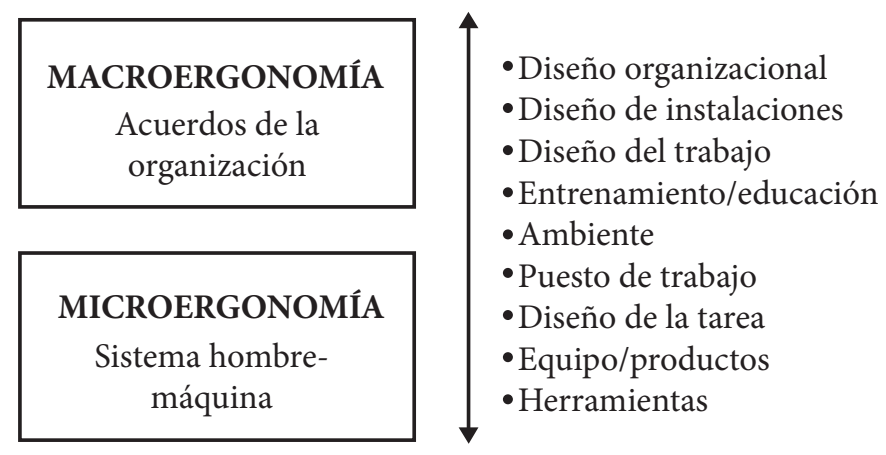

Figura 1. Aplicaciones de la Ergonomía

Fuente: adaptado de "Ergonomics guidelines for occupational health practice in industrially developing countries IAE”, de McPhee et al, 2010.

La macroergonomía, definida por Hendrick, muestra el "acercamiento socio-técnico y sistemático al diseño organizacional y sus formas de trabajo" (10) y busca la eficacia general del sistema, de manera que todos los subsistemas del lugar de trabajo estén coordinados, supervisados y realizados por cada trabajador que lleva a cabo una labor dentro del sistema más amplio (9). La microergonomía, concebida como "el estudio de la interacción hombre-máquina” (9, p.14), se centra en el trabajo con máquinas y herramientas, donde cualquier incompatibilidad entre la tarea, requisitos y capacidades del trabajador puede resultar en una situación subóptima. Por muchos años, la ergonomía se ha desarrollado ampliamente en este aspecto, cuya aplicabilidad busca la trasformación del puesto para que se ajuste a las necesidades y requerimientos del trabajador y la tarea.

Realyvásquez Vargas et al (11) desarrollaron y validaron un Cuestionario de Compabilidad Macroergonómica (CCM) en español, creado para la evaluar la aplicación de prácticas ergonómicas con relación a los factores: capital humano, organización, tareas, herramientas y tecnología, y condiciones ambientales en los sistemas de manufactura, cuyo análisis factorial mostró un alfa de Cronbach de $>0,7$. Rodríguez et al. (12) desarrollaron un Modelo de Madurez Ergonómica que es diligenciado por las empresas y que luego fue aplicado por Rodríguez y Pérez (13) en 5 empresas del sector industrial. Dicho modelo evidenció los factores influyentes en la poca madurez ergonómica que los ubica en niveles bajos.

De esta forma, este estudio buscó desarrollar y validar un instrumento que permitiera conocer el grado de cultura ergonómica observada por el trabajador dentro de una organización. En cuanto a la presencia y control de estrategias de macroergonomía y microergonomía, esto permite al empleador reflexionar sobre la forma como el trabajador percibe las estrategias implementadas, reconoce de la gestión ergonómica y se compromete con el control de los peligros y riesgos ergonómicos presentes en su lugar de trabajo. 


\section{Materiales y métodos}

\section{Construcción del cuestionario}

Tras una revisión de la literatura, se realizó un constructo teórico cuyos pilares se fundamentaron en el modelo de Scott, Kogi y McPhee(9) y que contempla las dimensiones macroergonomía y microergonomía y los componentes diseño organizacional, diseño de instalaciones, entrenamiento/educación, puesto de trabajo, diseño de la tarea, equipos/productos y herramientas (ver Tabla 1). A partir de este, se elaboró un cuestionario de 27 puntos con respuestas tipo Likert. Las preguntas se perfilaron de manera que pudieran ser resueltas en cualquier contexto empresarial. Se generó un primer borrador con cada uno de los ítems del cuestionario, que fue revisado por cinco expertos en ergonomía, los cuales hicieron ajustes de pertinencia y adecuación.

Tabla 1. Transición de la dimensión al ítem.

\begin{tabular}{|c|c|c|c|}
\hline Dimensiones & Componentes & Subcomponentes & Ítem \\
\hline \multirow{9}{*}{ 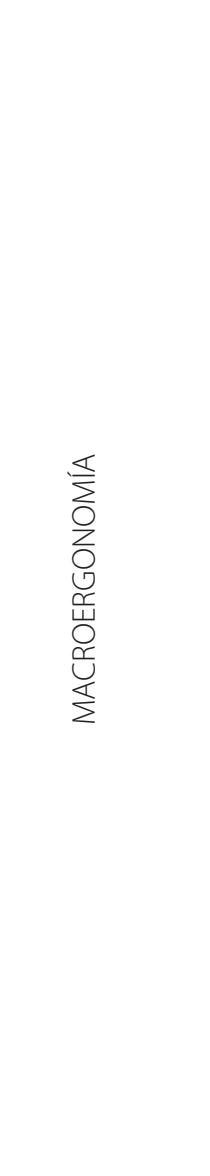 } & $\begin{array}{l}\text { Diseño } \\
\text { organizacional }\end{array}$ & $\begin{array}{l}\text { Ritmo y tiempos de } \\
\text { trabajo }\end{array}$ & $\begin{array}{l}\text { Organización de los turnos de } \\
\text { trabajo. }\end{array}$ \\
\hline & \multirow{5}{*}{$\begin{array}{l}\text { Diseño de } \\
\text { instalaciones }\end{array}$} & & Inspecciones al área de trabajo. \\
\hline & & Ambiente & $\begin{array}{l}\text { Mejoramiento de las } \\
\text { condiciones ambientales del } \\
\text { puesto de trabajo. }\end{array}$ \\
\hline & & Antropometría & $\begin{array}{l}\text { Mediciones antropométricas } \\
\text { con respecto a las zonas de } \\
\text { trabajo. }\end{array}$ \\
\hline & & Variedad de la tarea & $\begin{array}{l}\text { Desarrollo de distintas } \\
\text { habilidades en la labor } \\
\text { realizada. }\end{array}$ \\
\hline & & $\begin{array}{l}\text { Identidad de la } \\
\text { tarea }\end{array}$ & $\begin{array}{l}\text { Organización de labores a fin } \\
\text { de reconocer el producto de la } \\
\text { misma. }\end{array}$ \\
\hline & \multirow[t]{3}{*}{$\begin{array}{l}\text { Diseño del } \\
\text { trabajo }\end{array}$} & Valor de la tarea & $\begin{array}{l}\text { Organización de las labores } \\
\text { a fin de generar un producto } \\
\text { que sirva de insumo para otras } \\
\text { dependencias. }\end{array}$ \\
\hline & & Autonomía & $\begin{array}{l}\text { Facultad para tomar decisiones } \\
\text { en caso de dificultades en el } \\
\text { desarrollo de la labor. }\end{array}$ \\
\hline & & Retroalimentación & $\begin{array}{l}\text { Organización del trabajo de } \\
\text { manera que le permite obtener } \\
\text { información de los resultados } \\
\text { de la labor realizada. }\end{array}$ \\
\hline
\end{tabular}


Continuación Tabla 1. Transición de la dimensión al ítem

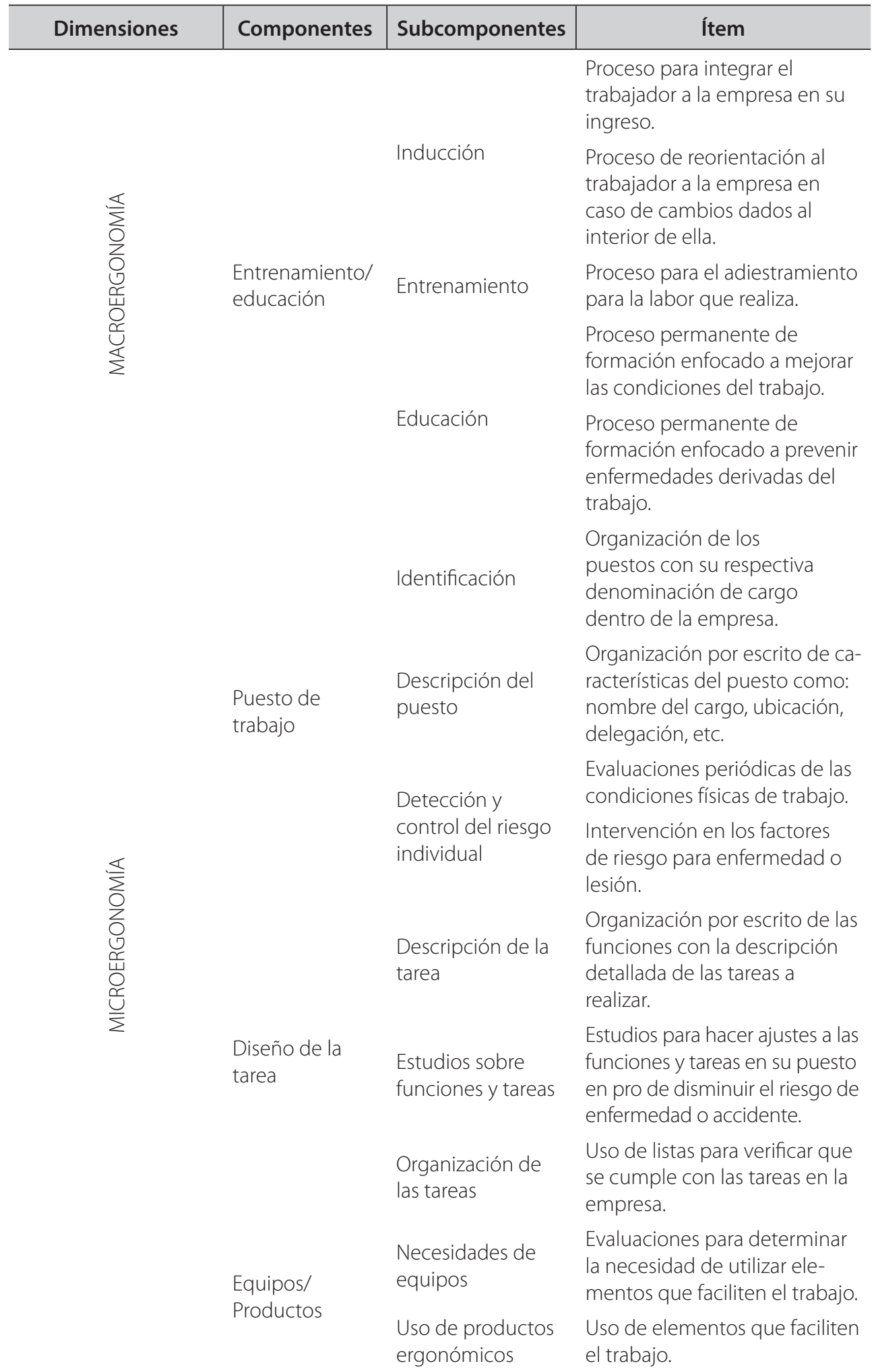


INVESTIGACIONES ANDINA No. 37, Vol. 20

Continuación Tabla 1. Transición de la dimensión al ítem.

\begin{tabular}{|c|c|c|c|}
\hline Dimensiones & Componentes & Subcomponentes & Ítem \\
\hline \multirow{3}{*}{ 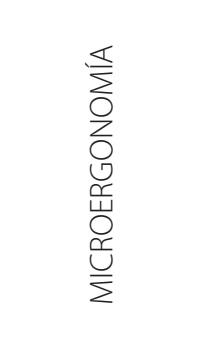 } & \multirow{3}{*}{ Herramientas } & & Seguridad de herramientas. \\
\hline & & Seguridad y confort & $\begin{array}{l}\text { Chequeos permanentes de la } \\
\text { función de las herramientas } \\
\text { usadas en el puesto de trabajo }\end{array}$ \\
\hline & & $\begin{array}{l}\text { Uso de } \\
\text { herramientas } \\
\text { ergonómicas }\end{array}$ & $\begin{array}{l}\text { Uso de herramientas de } \\
\text { trabajo que se ajustan a las } \\
\text { partes de su cuerpo para hacer } \\
\text { menos esfuerzo. }\end{array}$ \\
\hline
\end{tabular}

\section{Asignación de valores}

Las categorías tomadas de la escala de Likert planteadas en el cuestionario llevan al evaluador a ponderar cada ítem. Así, el total de las respuestas totalmente de acuerdo se multiplican por uno; parcialmente de acuerdo, por dos; parcialmente en desacuerdo, por tres; y totalmente en desacuerdo, por cuatro. Para llevar el total a una clasificación, a partir de los resultados se tomó el total de la sumatoria de respuestas de cada una de las cuatro categorías, y se trasladó a una tabla de ponderados que permiten, finalmente, dar el resultado del nivel en el que el trabajador clasifica a su empresa.

$\mathrm{Al}$ evaluar qué tanta cultura ergonómica se espera que una empresa pueda desarrollar, los autores han definido cuatro niveles de cultura ergonómica: baja, media, alta y muy alta, producto de la sumatoria de los totales parciales ya ponderados. Esta categorización permite orientar a la empresa sobre lo que consideran sus trabajadores en cuanto a actuaciones en ergonomía y comparar los resultados con lo existente (ver Tabla 2).

Tabla 2. Nivel de cultura ergonómica observado por el trabajador

\begin{tabular}{|c|c|}
\hline Nivel de Cultura Ergonómica & Interpretación \\
\hline $\begin{array}{l}\text { Baja } \\
\text { Puntaje entre } 79 \text { - } 104\end{array}$ & $\begin{array}{l}\text { El trabajador reconoce baja planeación y ejecución de } \\
\text { acciones en el desarrollo de una cultura ergonómica. }\end{array}$ \\
\hline $\begin{array}{l}\text { Media } \\
\text { Puntaje entre } 53 \text { - } 78\end{array}$ & $\begin{array}{l}\text { El trabajador reconoce algunos puntos importantes } \\
\text { que denotan un desarrollo incipiente de una cultura } \\
\text { ergonómica en la empresa. }\end{array}$ \\
\hline $\begin{array}{l}\text { Alta } \\
\text { Puntaje entre } 40-52\end{array}$ & $\begin{array}{l}\text { El trabajador reconoce acciones puntuales y } \\
\text { organizadas en el desarrollo de la cultura ergonómica } \\
\text { en la empresa. }\end{array}$ \\
\hline $\begin{array}{l}\text { Muy alta } \\
\text { Puntaje entre } 26-39\end{array}$ & $\begin{array}{l}\text { El trabajador reconoce un alto grado de desarrollo } \\
\text { de una cultura ergonómica en la empresa, lo que } \\
\text { demuestra un compromiso gerencial. }\end{array}$ \\
\hline
\end{tabular}




\section{Validez facial}

Se asignó la denominación definitiva del instrumento como: Cuestionario de Cultura Ergonómica en Centros de Trabajo CCE-T. Se aplicó a 16 trabajadores, seleccionados por conveniencia, cuyos aportes sugirieron cambios en aspectos de forma como el us de lenguaje común y general para ser aplicado a distintos sectores económicos. Igualmente, se determinó que este fuese auto-suministrado y que el tiempo aproximado de diligenciamiento estuviese alrededor de los 15 minutos.

\section{Validez y confiabilidad}

El proceso de validación se realizó entre junio de 2015 y diciembre de 2016. Se aplicó el CCE-T a una población de 162 trabajadores seleccionados a conveniencia, cuyos criterios de inclusión fueron ser mayores de 18 años y estar vinculados contractualmente a cualquier tipo de empresa por un tiempo igual o mayor a seis meses. Se realizó una solicitud de participación en el estudio a 12 empresas de los tres sectores; 10 aceptaron. Principalmente, se trataban de empresas del sector de servicios y el industrial. En todos los casos, se realizó el contacto mediante redes de contacto de los en- cuestadores colaboradores. Para la validación del CCE-T, se realizó un análisis factorial en el programa SPSS, versión 22.0. La versión final para validación estuvo compuesta por 27 ítems, cuya escala de respuesta fue: totalmente de acuerdo, parcialmente de acuerdo, parcialmente en desacuerdo y totalmente en desacuerdo.

\section{Consideraciones éticas}

El estudio se ciñó a los principios éticos de la Declaración de Helsinki y la Resolución 8430 de 1993 del Ministerio de Salud de Colombia, para así considerarlo sin riesgo. Se contó con la aprobación del Comité de Investigaciones de la Universidad Manuela Beltrán y la firma de un consentimiento informado por parte de los trabajadores.

\section{Resultados}

\section{Participantes}

La muestra $(\mathrm{n}=162)$ se conformó principalmente por hombres. Su edad promedio fue 35,5 años y registraron niveles de escolaridad altos. Llevaban en promedio 7 años laborando, la mayoría en horario diurno en empresas grandes (ver Tabla 3).

Tabla 3. Características sociodemográficas y ocupacionales de la población

\begin{tabular}{|c|c|c|c|}
\hline \multicolumn{2}{|c|}{$\begin{array}{l}\text { Características sociodemográficas } \\
\text { Estadística } n=162\end{array}$} & \multicolumn{2}{|c|}{$\begin{array}{c}\text { Características ocupacionales } \\
\text { Estadística } n=162\end{array}$} \\
\hline Edad & & Tiempo en & \\
\hline Media \pm DS & $35,5 \pm 11,42$ & Media \pm DS & $7,5 \pm 6,72$ \\
\hline Min - Max & $20-60$ & Min - Max & $1-36$ \\
\hline
\end{tabular}


Continuación Tabla 3. Características sociodemográficas y ocupacionales de la población.

\begin{tabular}{|c|c|c|c|}
\hline \multicolumn{2}{|c|}{$\begin{array}{l}\text { Características sociodemográficas } \\
\text { Estadística } n=162\end{array}$} & \multicolumn{2}{|c|}{$\begin{array}{l}\text { Características ocupacionales } \\
\text { Estadística } n=162\end{array}$} \\
\hline \multicolumn{2}{|l|}{ Edad agrupada $\mathrm{n}(\%)$} & \multicolumn{2}{|c|}{ Tiempo en el cargo agrupado n(\%) } \\
\hline 18-25 años & $29(17,9)$ & 1-5 años & $86(53,1)$ \\
\hline 26-35 años & $74(45,7)$ & 6-10 años & $31(19,1)$ \\
\hline 36-45 años & $24(14,8)$ & 11-15 años & $11(6,8)$ \\
\hline 46-55 años & $22(13,6)$ & 16-20 años & $32(19,8)$ \\
\hline \multirow[t]{2}{*}{ 56-65 años } & $13(8,0)$ & 26-30 años & $1(0,6)$ \\
\hline & & Mayor a 30 & $1(0,6)$ \\
\hline Sexo n(\%) & & Horario n(\%) & \\
\hline Masculino & $84(51,9)$ & Diurno & $136(184,0)$ \\
\hline \multirow[t]{2}{*}{ Femenino } & $78(48,1)$ & Nocturno & $2(1,2)$ \\
\hline & & Mixto & $24(14,8)$ \\
\hline Escolaridad n(\%) & & Tipo de empre & \\
\hline Primaria & $3(1,9)$ & Microempresa & $35(21,6)$ \\
\hline Secundaria & $29(17,9)$ & Pequeña & $11(6,8)$ \\
\hline Técnico/Tecnológico & $33(20,4)$ & Mediana & $39(24,1)$ \\
\hline Profesional & $47(29,0)$ & Grande & $77(47,5)$ \\
\hline Posgrado & $48(29,6)$ & & \\
\hline \multirow[t]{3}{*}{ Ninguna } & $2(1,2)$ & Sector de la ecc & \\
\hline & & Industrial & $72(44,4)$ \\
\hline & & Servicios & $90(55,6)$ \\
\hline
\end{tabular}

\section{Análisis de pertinencia y adecuación}

La validación de contenido, realizada por un panel de 5 expertos, permitió reconocer cambios en el uso de expresiones que pudiesen generar confusiones en quien aplica, así como determinar la pertinencia en la valoración de las dimensiones macroergonomía y microergonomía.

\section{Análisis factorial de la escala}

Para determinar los factores subyacentes en la escala, se realizó un análisis factorial por componentes principales.
Inicialmente, se realizó una prueba de Kaiser-Meyer-Olkin (KMO), que arrojó 0,804 como resultado. Este valor sugirió que la muestra era suficiente y meritoria para proseguir con el análisis factorial. Posteriormente, se realizó un análisis con los autovalores originales (ver Tabla 4). Se conservaron aquellos factores que cumplieron con los siguientes criterios: autovalor mayor de 1 (dado por la regla de Kaise-Gutman) y varianza explicada (mayor de 5\% para cada factor). Finalmente, se retuvieron cinco factores cuyos autovalores fueron mayores a 1 y que explicaron el 63,4\% de la varianza total. 
Tabla 4. Estadísticos de fiabilidad.

\begin{tabular}{c|c|c|c}
\hline Alpha de Cronbach & $\begin{array}{c}\text { Alpha de Cronbach } \\
\text { basada en los elementos } \\
\text { tipificados }\end{array}$ & N de elementos & Dimensión \\
\hline 0,896 & 0,895 & 26 & Total de elementos \\
0,772 & 0,771 & 14 & $\begin{array}{c}\text { Dimensión } \\
\text { macroergonomía } \\
0,862\end{array}$ \\
0,861 & 12 & $\begin{array}{c}\text { Dimensión } \\
\text { microergonomía }\end{array}$ \\
\hline
\end{tabular}

En la Tabla 5 se observa que el primer factor explica el 29,9\% de la varianza, el segundo factor explica cerca del 15\% y los demás factores explican menos del
$10 \%$ de la varianza cada uno. Además, los 3 primeros factores dan cuenta de más del $50 \%$ de la varianza de la escala.

Tabla 5. Análisis con autovalores originales del CCE-T.

\begin{tabular}{c|c|c|c|c}
\hline Factor & Autovalor & Diferencia & $\begin{array}{c}\text { Proporción de la } \\
\text { varianza }\end{array}$ & Varianza acumulada \\
\hline Factor 1 & 8,08 & 4,2 & 0,2995 & 0,2995 \\
Factor 2 & 3,87 & 1,73 & 0,1436 & 0,4431 \\
Factor 3 & 2,14 & 0,57 & 0,0794 & 0,5226 \\
Factor 4 & 1,56 & 0,12 & 0,0580 & 0,5805 \\
Factor 5 & 1.43 & 0,35 & 0,0533 & 0,6338 \\
\hline
\end{tabular}

Con estos cinco factores se obtuvo una matriz de componentes no rotada, con el fin de reagrupar los ítems para obterner menos factores y hacerlos lo más independientes posibles entre ellos. De acuerdo con lo anterior se obtuvieron dos factores, en donde la mayoría de ítems cargaron con valores superiores a 0,4 . Posteriormente, se realizó la rotación de la matriz obtenida. Se probó inicialmente una rotación ortogonal Va- rimax; sin embargo, no se obtuvo una estructura simple, dado que hay ítems que cargan en más de un factor, lo que hace que la matriz sea de más difícil de interpretar.

Por otra parte, se efectuó una rotación Promax. De igual manera, dicha rotación no arrojó una estructura fácil de interpretar. Por consiguiente, la matriz de datos sin rotar fue la que confirió 
una mejor estructura de los ítems y los factores. Para facilitar la interpretación de estos hallazgos, se eliminaron los valores con cargas menores de 0,40 . De acuerdo con lo anterior, el ítem número 15 fue el que menos carga presentó del total, dejando como resultado 26 ítems finales que componen el cuestionario.

\section{Análisis de Confiabilidad}

Para establecer la consistencia interna del CCE-T, se calculó el coeficiente alfa de Cronbach para toda la escala, que resultó en 0,90 , y para cada uno de los dominios que conforman la escala, los cuales corresponden a los conceptos de macroergonomía y microergonomía. En la Tabla 6 se incluyeron los principales coeficientes del análisis de confiabilidad; así mismo, se observó el comportamiento de cada ítem con respecto al total de la escala.

Tabla 6. Estadísticos totales elemento para las dimensiones macroergonomía y microergonomía

\begin{tabular}{|c|c|c|c|c|c}
\hline \multirow{2}{*}{ Ítem } & $\begin{array}{c}\text { Media de la } \\
\text { escala si se } \\
\text { elimina el } \\
\text { elemento }\end{array}$ & $\begin{array}{c}\text { Varianza de } \\
\text { la escala si } \\
\text { se elimina el } \\
\text { elemento }\end{array}$ & $\begin{array}{c}\text { Correlación } \\
\text { elemento- } \\
\text { total } \\
\text { corregida }\end{array}$ & $\begin{array}{c}\text { Correlación } \\
\text { múltiple al } \\
\text { cuadrado de }\end{array}$ & $\begin{array}{c}\text { Alfanbach } \\
\text { si se } \\
\text { elimina el } \\
\text { elemento }\end{array}$ \\
\hline
\end{tabular}

1. ¿Los turnos de trabajo están organizados según 26,33 44,720 , 245 , 428 , 770

las condiciones de la empresa?

2. ¿Se realizan inspecciones a las áreas donde se labora para tener un ambiente de trabajo confortable?

\section{Cuando se han} detectado problemas relacionados con el ambiente (por ejemplo: ruido excesivo, falta de iluminación, vibración, calor, humedad o frío extremo), ¿se han realizado los cambios pertinentes 
Continuación Tabla 6. Estadísticos totales elemento para las dimensiones macroergonomía y microergonomía

\begin{tabular}{c|c|c|c|c|c}
\hline \multirow{2}{*}{ Ítem } & $\begin{array}{c}\text { Media de la } \\
\text { escala si se } \\
\text { elimina el } \\
\text { elemento }\end{array}$ & $\begin{array}{c}\text { Varianza de } \\
\text { la escala si } \\
\text { se elimina el } \\
\text { elemento }\end{array}$ & $\begin{array}{c}\text { Correlación } \\
\text { elemento- } \\
\text { total } \\
\text { corregida }\end{array}$ & $\begin{array}{c}\text { Correlación } \\
\text { múltiple al } \\
\text { cuadrado }\end{array}$ & $\begin{array}{c}\text { Cronbach } \\
\text { si se } \\
\text { elimina el } \\
\text { elemento }\end{array}$ \\
\hline
\end{tabular}

4. ¿Se han realizado mediciones de las partes de su cuerpo para hacer ajustes a su puesto de trabajo durante el tiempo que lleva laborando en esta empresa?

5. ¿Se han hecho cambios para que en su labor usted desarrolle distintas habilidades y no se enfoque solamente en una?

\section{6. ¿La labor que} realiza en la empresa está organizada de manera que al realizar sus tareas de principio a fin, el resultado de lo que hace sea fácil de identificar?

7. ¿La labor que realiza genera un producto requerido por otras dependencias de la empresa?

8. ¿Su trabajo le permite ser autónomo en el momento de encontrarse con una dificultad en el desarrollo de sus tareas?

$\begin{array}{llll}25,05 & \text { 27 } 225 & \text {, 276 }\end{array}$

$\begin{array}{lllll}25,63 & 39,800 & 391 & , 394\end{array}$

$\begin{array}{lllll}26,02 & 428 & , 378 & , 460\end{array}$

$\begin{array}{llll}26,22 & \text {, 205 }\end{array}$

$\begin{array}{lllll}25,85 & \text {, 204 }\end{array}$


Continuación Tabla 6. Estadísticos totales elemento para las dimensiones macroergonomía y microergonomía

\begin{tabular}{|c|c|c|c|c|c|}
\hline Ítem & $\begin{array}{l}\text { Media de la } \\
\text { escala si se } \\
\text { elimina el } \\
\text { elemento }\end{array}$ & $\begin{array}{c}\text { Varianza de } \\
\text { la escala si } \\
\text { se elimina el } \\
\text { elemento }\end{array}$ & $\begin{array}{l}\text { Correlación } \\
\text { elemento- } \\
\text { total } \\
\text { corregida }\end{array}$ & $\begin{array}{l}\text { Correlación } \\
\text { múltiple al } \\
\text { cuadrado }\end{array}$ & $\begin{array}{c}\text { Alfa de } \\
\text { Cronbach } \\
\text { si se } \\
\text { elimina el } \\
\text { elemento }\end{array}$ \\
\hline $\begin{array}{l}\text { 9. ¿El trabajo } \\
\text { que realiza le } \\
\text { permite obtener } \\
\text { información de los } \\
\text { resultados de lo } \\
\text { que usted realiza? }\end{array}$ & 26,10 & 44,293 & ,161 & ,654 & ,777 \\
\hline $\begin{array}{l}\text { 10. Cuando ingre- } \\
\text { só a la empresa, } \\
\text { ¿Le brindaron } \\
\text { una inducción } \\
\text { completa sobre las } \\
\text { actividades que } \\
\text { debe realizar? }\end{array}$ & 26,21 & 40,465 &, 528 &, 551 & ,747 \\
\hline $\begin{array}{l}\text { 11. ¿Ha recibido } \\
\text { reinducción de la } \\
\text { labor que realiza? }\end{array}$ & 25,64 & 39,163 &, 445 &, 545 & ,753 \\
\hline $\begin{array}{l}\text { 12. ¿Recibe } \\
\text { entrenamiento } \\
\text { para mejorar la } \\
\text { labor que realiza? }\end{array}$ & 25,52 & 38,524 &, 558 & ,630 & ,741 \\
\hline $\begin{array}{l}\text { 13. ¿Se realizan jor- } \\
\text { nadas educativas } \\
\text { programadas para } \\
\text { mejorar las condi- } \\
\text { ciones del trabajo } \\
\text { que realiza? }\end{array}$ & 25,67 & 37,402 &, 570 & ,567 & ,738 \\
\hline $\begin{array}{l}\text { 14. ¿Se realizan } \\
\text { jornadas educati- } \\
\text { vas programadas } \\
\text { para enseñarle a } \\
\text { prevenir enferme- } \\
\text { dades derivadas } \\
\text { del trabajo que } \\
\text { realiza? }\end{array}$ & 25,17 & 39,767 &, 427 &, 412 & ,755 \\
\hline $\begin{array}{l}\text { 15. ¿Su lugar } \\
\text { de trabajo está } \\
\text { identificado con } \\
\text { el nombre de su } \\
\text { cargo dentro de la } \\
\text { empresa? }\end{array}$ & 24,48 & 52,773 &, 326 & ,397 & 863 \\
\hline
\end{tabular}


Continuación Tabla 6. Estadísticos totales elemento para las dimensiones macroergonomía y microergonomía

\begin{tabular}{|c|c|c|c|c|c}
\hline \multirow{2}{*}{ Ítem } & $\begin{array}{c}\text { Media de la } \\
\text { escala si se } \\
\text { elimina el } \\
\text { elemento }\end{array}$ & $\begin{array}{c}\text { Varianza de } \\
\text { la escala si } \\
\text { se elimina el } \\
\text { elemento }\end{array}$ & $\begin{array}{c}\text { Correlación } \\
\text { elemento- } \\
\text { total } \\
\text { corregida }\end{array}$ & $\begin{array}{c}\text { Correlación } \\
\text { múltiple al } \\
\text { cuadrado }\end{array}$ & $\begin{array}{c}\text { Cronbach } \\
\text { si se } \\
\text { elimina el } \\
\text { elemento }\end{array}$ \\
\hline
\end{tabular}

16. ¿Existe un

documento escrito

en la empresa

que registre todo

lo que incluye su

puesto de trabajo

$$
23,64
$$

$$
\text { 48,293 }
$$

, 466

, 499

(por ejemplo:

nombre del cargo,

funciones, etc.)?

17. ¿Se realizan

evaluaciones de

las condiciones

en las que se en-

cuentra su espacio

físico de trabajo?

18. ¿Se han hecho cambios en las tareas que usted realiza de manera que le generen menos riesgo de enfermedad o

lesión?

19. ¿Su labor tiene identificadas las funciones con la descripción deta-

llada de las tareas a realizar?

20. ¿Se han hecho ajustes a las

funciones y tareas en su puesto en pro de disminuir el riesgo de enfermedad o accidente?

21. ¿Utiliza listas para verificar que se cumple con 
Continuación Tabla 6. Estadísticos totales elemento para las dimensiones macroergonomía y microergonomía

\begin{tabular}{|c|c|c|c|c|c|}
\hline Ítem & $\begin{array}{l}\text { Media de la } \\
\text { escala si se } \\
\text { elimina el } \\
\text { elemento }\end{array}$ & $\begin{array}{c}\text { Varianza de } \\
\text { la escala si } \\
\text { se elimina el } \\
\text { elemento }\end{array}$ & $\begin{array}{l}\text { Correlación } \\
\text { elemento- } \\
\text { total } \\
\text { corregida }\end{array}$ & $\begin{array}{l}\text { Correlación } \\
\text { múltiple al } \\
\text { cuadrado }\end{array}$ & $\begin{array}{c}\text { Alfa de } \\
\text { Cronbach } \\
\text { si se } \\
\text { elimina el } \\
\text { elemento }\end{array}$ \\
\hline $\begin{array}{l}\text { 22. ¿Se han hecho } \\
\text { evaluaciones para } \\
\text { determinar la ne- } \\
\text { cesidad de utilizar } \\
\text { elementos que } \\
\text { faciliten el trabajo } \\
\text { que usted realiza? } \\
\text { (por ejemplo: apo- } \\
\text { ya-pies, apoya-mu- } \\
\text { ñecas, soportes } \\
\text { para columna } \\
\text { vertebral, descan- } \\
\text { sa-pies, soporte } \\
\text { para portátiles, etc.) }\end{array}$ & 23,59 & 46,404 &, 576 &, 599 & 849 \\
\hline $\begin{array}{l}\text { 23. ¿Utilizan ele- } \\
\text { mentos que facili- } \\
\text { ten el trabajo que } \\
\text { usted realiza? (por } \\
\text { ejemplo: apoya- } \\
\text { piés, apoya-muñe- } \\
\text { cas, soportes para } \\
\text { columna vertebral, } \\
\text { descansa-pies, } \\
\text { soporte para por- } \\
\text { tátiles, etc.) }\end{array}$ & 23,53 & 48,015 &, 549 & ,502 &, 850 \\
\hline $\begin{array}{l}\text { 24. ¿Considera } \\
\text { seguras las herra- } \\
\text { mientas usadas } \\
\text { para su labor? }\end{array}$ & 24,41 & 52,791 & ,386 &, 448 & 860 \\
\hline $\begin{array}{l}\text { 25. ¿Se hacen che- } \\
\text { queos permanen- } \\
\text { tes de la función } \\
\text { de las herramien- } \\
\text { tas usadas en su } \\
\text { puesto de trabajo? }\end{array}$ & 23,84 & 49,080 & ,605 &, 448 & 848 \\
\hline $\begin{array}{l}\text { 26. ¿Sus he- } \\
\text { rramientas de } \\
\text { trabajo se ajustan } \\
\text { a las partes de su } \\
\text { cuerpo para que } \\
\text { usted haga menos } \\
\text { esfuerzo? }\end{array}$ & 23,51 & 46,773 & ,637 & 640 & 844 \\
\hline
\end{tabular}


Con la eliminación de la pregunta 15, el cuestionario, en general, tiene un alfa de Cronbach de 0,901. La dimensión macroergonomía observa un alfa de Cronbach alto $(0,772)$, mientras la dimensión microergonomía observa un alfa de
Cronbach muy alto $(0,874)$. El perfil de cultura ergonómica que se observa en los sujetos de estudio es de nivel medio, del sector industrial y de empresas grandes, principalmente (ver tabla 7).

Tabla 7. Nivel de cultura ergonómica

\begin{tabular}{|c|c|c|c|c|}
\hline \multicolumn{5}{|c|}{$\begin{array}{l}\text { Nivel de Cultura Ergonómica } \\
\text { Estadística } n=162\end{array}$} \\
\hline \multicolumn{2}{|c|}{ Nivel de Cultura Ergonómica n(\%) } & \multicolumn{3}{|c|}{$\begin{array}{l}\text { Nivel de Cultura Ergonómica * } \\
\text { Sector empresa n (\%) }\end{array}$} \\
\hline Muy Alta & $0 \quad(0,0)$ & & Industrial & Servicios \\
\hline Alta & $7 \quad(4,3)$ & Muy Alta & $0 \quad(0,0)$ & $0 \quad(0,0)$ \\
\hline Media & $92(56,8)$ & Alta & $2(28,6)$ & $5 \quad(71,4)$ \\
\hline \multirow[t]{5}{*}{ Baja } & $63(38,9)$ & Media & $43(46,7)$ & $49(53,3)$ \\
\hline & & Baja & $48(76,2)$ & $15(23,8)$ \\
\hline & & Total & $93(57,4)$ & $69(42,6)$ \\
\hline & \multicolumn{3}{|c|}{$\begin{array}{l}\text { Nivel de Cultura Ergonómica } \\
\text { * Tipo de empresa n(\%) }\end{array}$} & \\
\hline & Micro & Pequeña & Mediana & Grande \\
\hline Muy Alta & $0 \quad(0,0)$ & $0 \quad(0,0)$ & $0 \quad(0,0)$ & $0 \quad(0,0)$ \\
\hline Alta & $4 \quad(57,1)$ & $1(14,3)$ & $2(28,6)$ & $0 \quad(0,0)$ \\
\hline Media & $16(17,4)$ & $4 \quad(4,3)$ & $25(27,2)$ & $47(51,1)$ \\
\hline Baja & $15(23,8)$ & $6(9,5)$ & $12(19,0)$ & $30(47,6)$ \\
\hline Total & $35(21,6)$ & $11(6,8)$ & $39(24,1)$ & $77(47,5)$ \\
\hline
\end{tabular}

\section{Discusión}

El CCE-T definitivo cuenta con un encabezado de características sociodemográficas y ocupacionales, y 26 ítems que evalúan acciones en las dos dimensiones. Según la literatura disponible, las escalas similares a la presentada en este artícu- lo son difíciles de encontrar, debido al concepto tan heterogéneo de ergonomía entre los trabajadores. Además, es posible que algunos instrumentos sean muy generales y carezcan de especificidad o profundidad. Así mismo, es posible que otros instrumentos tengan debilidades en cuanto a limitaciones psicométricas. 
Real y Vásquez et al. (11) estudiaron a profundidad la dimensión macroergonómica en el CCM. El elemento relacionado con turnos de trabajo en el CCE-T mostró un alfa significativo (0,770), mientras que en el CCM, al preguntar por la comodidad en los horarios, se observa un alfa bajo $(0,287)$. Si bien la mayoría de las empresas, de una u otra forma, implementan turnos, también los establecen en horarios irregulares. En consecuencia, son las empresas las que deben poner mayor atención a esta forma de organización del trabajo, por sus efectos en la salud de los trabajadores (12), pues se ha estudiado la probabilidad de desarrollar cáncer en personas que trabajan por turnos con interrupciones del ritmo circadiano (13).

Las inspecciones a las áreas, equipos e instalaciones buscan identificar el mayor número de condiciones subestándar en el lugar de trabajo (14) que puedan representar riesgo para su seguridad y salud. Cuando una empresa implementa inspecciones planeadas (sistemáticas), obtiene herramientas para el control de los peligros y riesgos a corto, mediano y largo plazo. Real y Vásquez et al. (11) hallaron un alfa significativo con respecto a la importancia que dan los trabajadores a la realización de inspecciones, similar a lo observado en el CCT-E.

El diseño de instalaciones incluye la evaluación de las condiciones ambientales del lugar de trabajo y el desarrollo de subprogramas de higiene ocupacional busca preservar la integridad física y mental mediante la implantación de normas, métodos y sistemas para prevenir accidentes, disminuir la causa de enfermedades y crear condiciones de trabajo confortables (15). El CCE-T muestra un alfa significativo en este ítem, al igual que los descritos por Real y Vásquez et al. (12) en el CCM. En aspectos como la antropometría, ciencia indispensable para el diseño del trabajo, los dos cuestionarios reflejan ser consistentes, ya que preguntan al trabajador sobre su aplicación. La implementación de esta en las empresas ayuda a prevenir situaciones desfavorables, reduciendo la probabilidad de sufrir un DME asociado a la labor (16) (17).

Las empresas deben implementar también condiciones que promuevan aspectos como la variedad de la tarea, presente cuando se desarrollan distintas habilidades en la labor, la identidad de la tarea, que se proyecta como la organización de labores a fin de reconocer el producto de la misma, y el valor de la tarea, que tiene en cuenta la organización de las labores con miras a generar un producto que sirva de insumo para otras dependencias. Real y Vásquez et al. (11) mostraron que estos aspectos no resultaron consistentes en su cuestionario, a diferencia del CCE-T que muestra un alfa de 0,759 en este ítem.

Aspectos relacionados con la autonomía, expresada como la facultad para tomar decisiones en caso de dificultades en el desarrollo de la labor, fueron consistentes en el CCE-T y en el CCM. El entrenamiento y la educación para la labor enriquece los procesos de integración al trabajador, así como su reorientación, adiestramiento y formación, pues impactan en las condiciones de trabajo y en la prevención 
de enfermedades. Las empresas deben también implementar la evaluación de dichas condiciones e intervenir en los factores definidos como estrategia preventiva. En el estudio de Real y Vásquez et al. (11), este aspecto tuvo un alfa bajo $(0,444)$ mientras que en el CCE-T el alfa superó los 0,70 .

Una empresa con cultura ergonómica debe organizar las funciones de cada puesto, igualmente, estudiarlas y ajustarlas en aras de disminuir el riesgo por enfermedad o accidente. Actualmente, es de vital importancia llevar a cabo evaluaciones periódicas de las condiciones físicas de trabajo; las empresas deben realizar intervenciones en los factores de riesgo para enfermedad o lesión. Este aspecto se hace complejo de comparar, pues mientras el CCE-T pregunta al trabajador si se evalúan aspectos del espacio físico de trabajo, el CCM lo hace por observación. A este respecto, es importante destacar que los métodos muchas veces son tan específicos que la evaluación se torna dispendiosa, razón por la que la creación y validación de métodos integrales se vuelve indispensable, principalmente para los peligros por posturas y movimientos forzados (18).

El hecho de que los trabajadores califiquen sus empresas en niveles inferiores debe permitir un análisis por parte de estas, pues no siempre la forma como llega la información sobre procedimientos se observada como actividades dentro de lo que se considera el sistema de gestión de salud y seguridad en el trabajo. El estudio pretendió considerar a todo tipo de trabajador como elemento idóneo para validarlo; sin embargo, por razones de azar, la mayoría de los trabajadores pertenecían al sector de industria y servicios, por lo que se sugiere aplicar el cuestionario en una segunda oportunidad, en distintas poblaciones, para establecer las diferencias y comportamientos del mismo en todos los sectores económicos.

\section{Conclusiones}

La mayoría de las preguntas formuladas en el cuestionario presentan un alfa de Cronbach alto o muy alto. Esto, a la luz de los resultados, denota fiabilidad de sus ítems, cuyos ajustes de forma incorporados, fueron de gran impacto en los resultados. Como fortaleza del CCE-T, los evaluadores reconocieron como importante el hecho de que la herramienta es ágil al momento de dar la respuesta y consideraron importantes los ítems abordados. La valoración a partir del uso del CCE-T permite dar una idea de lo que el trabajador observa sobre el desarrollo y aplicación de la ergonomía en su empresa. Por consiguiente, se considera un elemento valioso como mecanismo retroalimentador de procesos en salud colectiva y como herramienta diagnóstica.

\section{Agradecimientos}

Los autores expresan su agradecimiento a la fisioterapeuta Sandra Juliana Sanabria Sarmiento y a los estudiantes de la Especialización en Salud Ocupacional y Riesgos Laborales de la Universidad Manuela Beltrán, Seccional Bucaramanga, por su apoyo en la realización de este estudio. 


\section{Descargos de responsabilidad}

Todos los datos y comentarios plasmados en este artículo son responsabilidad de los autores. Este estudio fue financiado en su totalidad por la Universidad Manuela Beltrán, Seccional Bucaramanga. Los autores declaran no poseer conflictos de interés.

\section{Referencias}

1. Nunes I. Introduction to musculoskeletal disorders. 2017. [citado 2018, junio, 3]. En:OSH-WIKI: networking knowledge. [Internet]. EU-OSHA. Disponible en: https:// oshwiki.eu/wiki/Introduction_to_ musculoskeletal_disorders

2. Health and Safety Executive. Work Related Musculoskeletal Disorder (WRMSDs) Statistics, Great Britain 2017. [Internet]. Liverpool, UK: Health and Safety Executive; 2016. [citado 2018, enero 30]. 22 p. Disponible en: http://www.hse.gov.uk/statistics/ causdis/musculoskeletal/msd.pdf

3. Minister of Employment and Labor. Industrial Disaster in Korea. Minister of Employment and Labor; Sejong, Korea: 2017.

4. Morken T., et al. Low back pain and widespread pain predict sickness absence among industrial workers. BMC Musculoskelet Disord. [Internet]. 2003;4:21. [citado 2018, febrero, 4]. Disponible en: $\mathrm{h} \mathrm{t} \mathrm{t}$ ps: / / b mcmusculoskeletdi-s ord.biomedcentral. com/tra c k /pdf/10.1186/1471-2474-4-21

5. Ministerio del Trabajo de Colombia. Segunda encuesta nacional de condiciones de seguridad y salud en el trabajo en el sistema de riesgos laborales. Bogotá: Ministerio del trabajo; 2013. 56 p.

6. Sultan-Taïeb H., et al. Economic evaluations of ergonomic interventions pre- disorders: a systematic review of organizational-level interventions. BMC public health. [Internet]. 2017;17:935 [citado 2018, junio, 22]. Disponible en: https:// bmcpublichealth.biomedcentral.com/ articles/10.1186/s12889-017-4935-y

7. Cadavid Guzman E., Saenz Zapata LM. La ergonomía en el contexto de la prevención. Realidad y propuestas educativas. Memorias: 16a Semana de la Salud Ocupacional y 90 Congreso Colombiano de Ergonomía. Medellín (Colombia). 10 de noviembre de 2010.

8. Gómez-Conesa A., MartínezGonzález M. Ergonomía. Historia y ámbitos de aplicación, Fisioterapia. 2002;24(1):3-10.

9. Scott P., Kogi K., McPhee B. Ergonomics guidelines for occupational health practice in industrially developing countries. Darmstadt: International Ergonomics Association (IEA), International Commission on Occupational Health (ICOH); 2010. $100 \mathrm{p}$.

10. Hendrick, $H$. The technology of ergonomics, Theor Issues Ergon Sci. 2000;1(1):22-33.

11. Realyvásquez A., García J., Blanco J. Desarrollo y validación de un cuestionario de compatibilidad macroergonómica. Contaduría y Administración. 2016;61(3):478-98. 
12. World Health Organization: International Agency for Research on Cancer. Painting, firefighting, and shiftwork. IARC Monographs on the Evaluation of Carcinogenic Risks to Humans. 2010;98.

13. Haus E., Smolensky M. Shift work and cancer risk: potential mechanistic roles of circadian disruption, light at night, and sleep deprivation. Sleep Med Rev. 2013;17(4):273-84.

14. Instituto Colombiano de Normas Técnicas y Certificación. Norma Técnica CoIombiana 4114. Bogotá: ICONTEC; 1997. $19 \mathrm{p}$.
15. Meza S. Higiene y Seguridad Industrial. México, D.F.: Instituto Politécnico Nacional; 1998. 94 p.

16. Obregón M. Fundamentos de Ergonomía. Grupo Editorial Patria; 2016. 353 p.

17. Nava R., Castro J., Rojas L., Gómez $M$. Evaluación ergonómica de los puestos de trabajo del área administrativa. Redieluz. 2013;3(1-2):27-35.

18. López B., González E., Colunga C., Oliva E. Evaluación de sobrecarga postural en trabajadores: revisión de la literatura. Ciencia \& Trabajo. 2014;16(50):111-5. 
INVESTIGACIONES ANDINA No. 37, Vol. 20 